\title{
Functional Heterogeneity of Retinal Dopaminergic Neurons Underlying Their Multiple Roles in Vision
}

\author{
Dao-Qi Zhang, Tong-Rong Zhou, and Douglas G. McMahon \\ Department of Biological Sciences, Vanderbilt University, Nashville, Tennessee 37235
}

\begin{abstract}
Dopaminergic neurons play key roles in the CNS, mediating basic mechanisms of vision, movement, motivation, and mood. The most accessible dopaminergic neurons of the vertebrate CNS are the dopaminergic amacrine cells of the retina. Here, we have characterized the intrinsic neural activity, synaptic input, and light responses of retinal dopaminergic neurons in situ, using targeted electrophysiological recordings of fluorescent neurons in TH::RFP (tyrosine hydroxylase gene promoter::red fluorescent protein) transgenic mice. Dopaminergic amacrine cells exhibit two classes of intrinsic bursting in the dark, shaped by inhibitory synaptic inputs, and two classes of light responses, $\mathrm{ON}$-transient and $\mathrm{ON}$-sustained, as well as light-independent activity, tuned to mediate specific dopaminergic functions in vision. The functional heterogeneity revealed in dopaminergic amacrine cells provides a cellular basis for the multiple roles of dopaminergic amacrine neurons in vision and is likely a general property of dopaminergic neurons throughout the CNS.
\end{abstract}

Key words: retina; dopamine; electrophysiology; transgene; light response; mouse

\section{Introduction}

Dopamine, released by specific populations of dopaminergic neurons, is a key modulatory neurotransmitter in the CNS, mediating sensory adaptation, motor program initiation, and reward behaviors. In addition, dysregulation of dopaminergic function plays a critical role in a range of neurological disorders such as parkinsonism, addiction, and schizophrenia. The most accessible dopaminergic neurons of the vertebrate CNS are the dopaminergic amacrine cells (DA cells) of the retina. Retinal dopamine has multiple roles in vision, mediating visual processes such as adapting light/dark retinal circuits, serving as an output of the retinal circadian clock, and influencing trophic processes, including photoreceptor survival and the degree of refractive errors in myopia (Witkovsky, 2004).

The frequency and pattern of dopaminergic neuron electrical activity (spiking) is a key factor regulating the secretion of dopamine (Gonon, 1988; Puopolo et al., 2001; Floresco et al., 2003). Isolated retinal DA cells exhibit spontaneous spiking and dopamine release (Feigenspan et al., 1998; Puopolo et al., 2001), but less is known about the cellular mechanisms by which these neurons are regulated in intact neural circuits by relevant stimuli. Despite the description of retinal DA cells 3 decades ago (Dowling and Ehinger, 1975) and the importance of retinal dopamine for visual function, the regulation of retinal dopamine secretion has remained unclear. Experiments attempting to define the stimulus conditions for dopamine release have yielded widely varying results suggesting that dopamine secretion can be stimulated by prolonged darkness or steady and flickering illumina-

Received 0ct. 13, 2006; revised Dec. 11, 2006; accepted Dec. 12, 2006.

This work was supported by National Institutes of Health Grant R01 EY15815 to D.G.M.

Correspondence should be addressed to Douglas G. McMahon, Department of Biological Sciences, Vanderbilt

University, VUStation B, P.0. Box35-1634, Nashville, TN37235-1634. E-mail:douglas.g.mcmahon@vanderbilt.edu. DOI:10.1523/JNEUROSCI.4478-06.2007

Copyright $\odot 2007$ Society for Neuroscience $\quad$ 0270-6474/07/270692-08\$15.00/0 tion, and it can also be mediated by the retinal circadian clock in a light-independent manner (Bauer et al., 1980; Mangel and Dowling, 1985; Umino et al., 1991; Weiler et al., 1997; Nir et al., 2000; Doyle et al., 2002a,b; Ribelayga et al., 2004).

Here, we have used a transgenic mouse model in which catecholaminergic neurons are marked with red fluorescent protein (RFP) driven by the tyrosine hydroxylase $(\mathrm{TH})$ gene promoter (TH::RFP) (Zhang et al., 2004) to target retinal dopaminergic amacrine cells in the intact retina for electrophysiological analysis. We have examined the regulation of retinal DA cells by synaptic input and light stimuli and now report that, in situ, DA cells exhibit two classes of spontaneous bursting that are influenced by glycinergic and GABAergic inhibition and, in addition, exhibit light-independent activity, as well as two distinct classes of light responses, ON-transient and ON-sustained. This surprising functional heterogeneity of retinal DA cells provides a basis on which to unify previous results regarding the multiple roles of retinal dopamine in visual function and is likely a general property of dopaminergic neurons throughout the CNS.

\section{Materials and Methods}

Animals. A transgenic mouse model expressing RFP under the control of the TH promoter (Zhang et al., 2004) was used for DA cell recordings. At the time of the experiments, the original line was backcrossed to C57BL/6J (The Jackson Laboratory, Bar Harbor, ME) for five to six generations. Adult mice used in this study were hemizygous for the TH::RFP transgene. All procedures conformed to National Institutes of Health and Vanderbilt University animal care and use guidelines. Mice were maintained on a $12 \mathrm{~h}$ light/dark cycle with lights on at 7 A.M. central standard time.

Tissue preparation. To minimize light exposure of retinas, all dissection procedures were performed under infrared illumination using either night-vision goggles or dual-unit Nitemare infrared scopes (BE Meyers, Redmond, WA). Mice were dark adapted for $1-2 \mathrm{~h}$ before the experiments. Mice were killed by cervical dislocation, and both eyes were re- 
moved. The eyecup was opened by an encircling cut along the ora serrata, and the retina was separated from the sclera in oxygenated extracellular medium. The extracellular solution contained the following (in mM): 125 $\mathrm{NaCl}, 2.5 \mathrm{KCl}, 1 \mathrm{MgSO}_{4}, 2 \mathrm{CaCl}_{2}, 1.25 \mathrm{NaH}_{2} \mathrm{PO}_{4}, 20$ glucose, and 26 $\mathrm{NaHCO}_{4}$, bubbled with a gaseous mixture of $95 \% \mathrm{O}_{2} / 5 \% \mathrm{CO}_{2}$. The whole-mount retina was placed photoreceptor-side down in the recording chamber. The chamber was then mounted on the stage of an upright conventional fluorescence microscope (Axioscope; Zeiss, Oberkochen, Germany) within a light-tight Faraday cage. Oxygenated medium was continuously perfused into the recording chamber at a rate of $\sim 2-3$ $\mathrm{ml} / \mathrm{min}$, and the superfusate was kept at $\sim 34^{\circ} \mathrm{C}$ by a temperature-control unit (TC-344A; Warner Instruments, Hamden, CT).

Electrophysiological recordings. All recordings were performed between Zeitgeber time 5 (ZT5) and ZT12 (ZT0, lights on; ZT12, lights off). The retina was maintained in darkness for $1 \mathrm{~h}$ before recording. Cells and recording pipettes were viewed on a video monitor coupled to a CCD camera (Princeton Instruments, Trenton, NJ) mounted on the microscope. TH::RFP-expressing cells were first identified by fluorescence microscopy using a DsRed filter set (41002c; Chroma Technology, Brattleboro, VT) with a brief "snap shot" of fluorescence excitation light (1-5s). Then the identified cells and glass electrode were visualized using infrared differential interference contrast (DIC) optics for patch-clamp recording.

Loose-patch voltage-clamp recordings were made from the soma of either DA cells or DiI-labeled ganglion cells using glass pipettes (A-M Systems, Carlsborg, WA) prepared using the DMZ universal puller (Zeitz-Instrumente, Munich, Germany). The pipettes were filled with a solution containing $150 \mathrm{~mm} \mathrm{NaCl}$ and $10 \mathrm{~mm}$ HEPES, with a pH of 7.5. The resistance of pipettes filled with this solution was 4-5 $\mathrm{M} \Omega$. Experiments were executed, and data were recorded using Clampex 8.0 software connected to an Axopatch-1D amplifier (Molecular Devices, Palo Alto, CA) via a Digidata 1322A digitizer (Molecular Devices). The holding potential within the pipette was $0 \mathrm{mV}$. Currents were low-pass filtered at $1 \mathrm{kHz}(-3 \mathrm{~dB}$ Bessel filter) and digitally sampled at $3.33 \mathrm{kHz}$.

L-(+)-2-4-Amino-4-phosphonobutyric acid (L-AP-4) and DL-threo$\beta$-benzyloxyaspartate (TBOA) were purchased from Tocris (Ellisville, $\mathrm{MO})$. All other drugs including $S(-)$-eticlopride hydrochloride, carbenoxolone, 2-(3-carboxypropyl)-3-amino-6-(4 methoxyphenyl) pyridazinium bromide (GABAzine), D(-)-2-amino-7-phosphonoheptanoic acid (AP-7), 6-cyano-7-nitroquinoxaline-2,3-dione disodium salt (CNQX), and imidazole-4-acetic acid sodium salt were purchased from Sigma (St. Louis, MO). All drugs were prepared as concentrated stock solutions and diluted to working concentrations in extracellular medium.

Light stimulation. Light stimuli were generated using a tricolor lightemitting diode (LED) lamp with 470, 525, and $630 \mathrm{~nm}$ wavelengths (Lighting Components, Brooklyn, NY). Uniform full-field illumination was delivered from the LED to the retina, via the microscope condenser. The LED was controlled by Clampex 8 software, and light intensity was adjusted by varying the output voltage from the software. Photon fluxes at the various wavelengths at the surface of the retina were measured with a Li-Cor (Lincoln, NE) quantum sensor (model Li-190SB) and radiometer/photometer (model Li-185B). The photon fluxes in the present study were $1.39 \times 10^{18}$ photons $/ \mathrm{m}^{2} / \mathrm{s}$ for $470 \mathrm{~nm}, 8.82 \times 10^{18}$ photons/ $\mathrm{m}^{2} / \mathrm{s}$ for $525 \mathrm{~nm}$, and $8.28 \times 10^{18}$ photons $/ \mathrm{m}^{2} / \mathrm{s}$ for $630 \mathrm{~nm}$ lights, all of which presumably saturate the rod responses and activate cones (Protti et al., 2005).

Data analysis. Data were analyzed using the Clampfit 9 (Molecular Devices) and SigmaPlot 10 (Systat Software, Erkrath, Germany) software packages. Firing rates and bursting activity were measured from $90 \mathrm{~s}$ recordings of each cell using the Event Detection program of the Clampfit 9 software. Generally, the interspike intervals within bursts are short, and spike amplitudes progressively decrease. The following criteria were used to detect bursts using the Event Analysis program of the Clampfit 9: at least three successive spikes within the burst and burst onset initiated by two consecutive spikes with an interspike interval $<80 \mathrm{~ms}$ and terminated with two spikes having an interspike interval $>160 \mathrm{~ms}$ (Grace and Bunney, 1984). We evaluated both burst quantity (percentage of spikes generated in bursts, percentage of time spent bursting, and burst rate) and burst quality (number of spikes per burst, burst duration, and frequency of spikes within bursts). To compare values from two conditions, paired $t$ tests were used. The level of significance was set at $p<0.05$. All values are given as the mean \pm SEM.

\section{Results}

Electrophysiological recordings were performed on $T H:$ RFPmarked DA cells in isolated whole-mount retinas. Two types of catecholaminergic amacrine cells express $T H$-driven RFP in the living retinas of $T H:: R F P$ transgenic mice, DA cells, and type 2 catecholaminergic amacrine cells, and these two classes of neurons are readily distinguished on visual inspection by their different soma sizes, location, and dendritic projections (Zhang et al., 2004). To minimize the light exposure of isolated retinas, retinas were prepared for recording under infrared illumination, and red fluorescent DA cells were localized by brief snap shots of imaging light and recorded using a cooled CCD camera (see Materials and Methods). Subsequent placement of recording electrodes was monitored using infrared optics. Although we did not quantify any effects on retinal adaptation of the fluorescent light used to locate cells, the retinas in these experiments were likely in a partially dark-adapted state, rather than a fully dark-adapted state, during data collection. A confocal fluorescence image of a DA cell in a living retinal whole mount, showing its characteristic large soma size and two to three thick dendritic branches, as well as an infrared DIC image of a DA neuron and the recording electrode, are shown in Figure $1 A$.

\section{Dopaminergic amacrine cells in situ exhibit spontaneous bursting}

Spike activity of DA cells was recorded extracellularly using loose-patch voltage-clamp recording from DA cell somata. In the dark, DA cells in situ exhibited patterns of spontaneous spike activity that were a mixture of bursting and irregular single spikes. These patterns were consistent and stable within cells, but the degree of bursting versus single spiking varied widely across cells. Figure 1, $B$ and $C$, shows spontaneous spike trains from DA neurons representing the extremes of this distribution of spike patterns. The neuron in Figure $1 B$ expressed $94 \%$ of its spikes within bursts, whereas the neuron in Figure $1 C$ expressed only $5 \%$ of its spikes within bursts. Analysis of the spiking patterns with a specific burst detection method (see Materials and Methods) demonstrated that 92 of 93 recorded cells exhibited some degree of bursting. Summary data on the bursting characteristics of DA cells is shown in Table 1 . The distribution of firing rates in DA cells was adequately described by a single broad distribution, but the distribution of spike patterns was not, containing distinct high-bursting and low-bursting cell populations (Fig. 1D). The mixed bursting/irregular single-spike patterns of spontaneous activity we observed in DA neurons in situ are distinct from the monotonic single-spike regular spontaneous activity observed in isolated dispersed DA cells (Feigenspan et al., 1998), suggesting regulation of DA neuron activity in situ by synaptic input.

\section{Synaptic inputs inhibit DA cell bursting}

The regulation of bursting in dopaminergic neurons is of particular interest, because bursting activity patterns are associated with increased dopamine release compared with single spiking (Gonon, 1988; Floresco et al., 2003). DA cells are postsynaptic to glutamatergic bipolar cells, GABAergic amacrine cells, and glycinergic AII amacrine cells and express a variety of ionotropic glutamate, $\mathrm{GABA}_{\mathrm{A}}$, and glycine receptors (Gustincich et al., 1997, 1999). Thus, we tested the ability of each of these synaptic inputs 

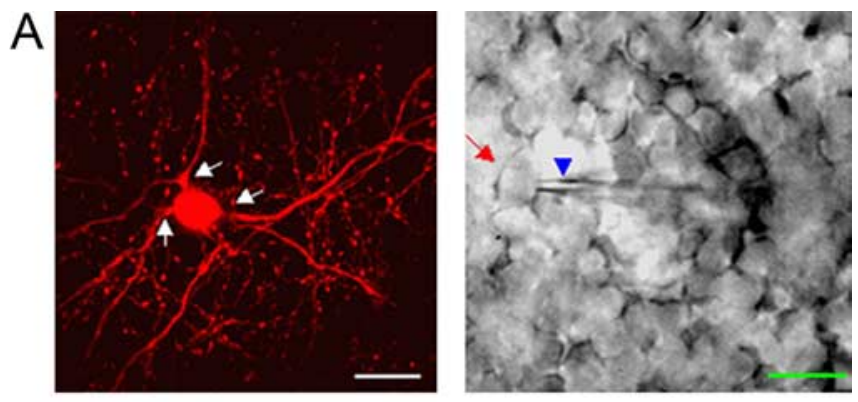

B

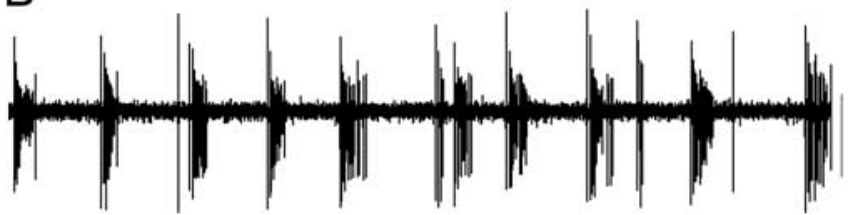

C

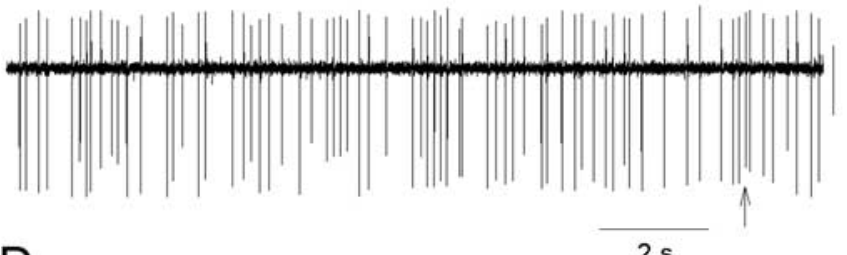

D

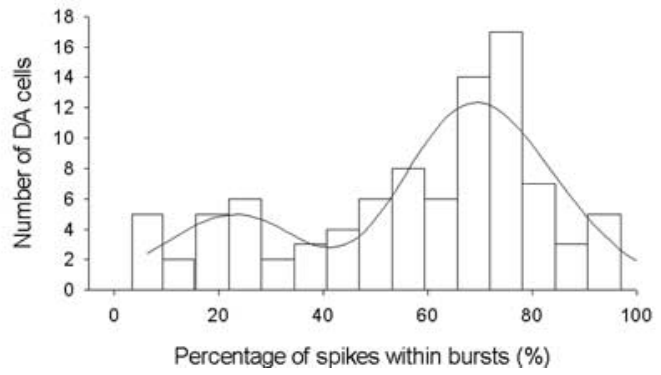

Figure 1. Spontaneous spiking/bursting of DA neurons in the dark. A, Fluorescence (left) and infrared (right) images of DA neurons in TH:RFP mouse retinal whole mounts illustrating cell targeting and recording. Arrows in the fluorescence photomicrograph point to the main processes of a DA cell. The arrow (red) and arrowhead (blue) in the infrared image point to the soma of the TH::RFP-expressing cell and the recording pipette, respectively. DA cells in situ exhibited mixed single-spike/bursting patterns. Scale bars, $20 \mu \mathrm{m}$. B illustrates a recording dominated by bursting (firing rate $10.9 \mathrm{~Hz}, 94 \%$ spikes in bursts), whereas $C$ is dominated by single-spike firing but also spikes containing bursts (arrow; firing rate of $4.6 \mathrm{~Hz}, 5 \%$ spikes in bursts). Loosepatch recordings were made using a voltage-clamp mode with the pipette holding a potential of $0 \mathrm{mV}$ in this figure, as well as in the following figures. Calibration: $40 \mathrm{pA}, 2 \mathrm{~s}$. D. Histogram of percentage of spikes within bursts. DA cell spike trains yielded a two-peaked Gaussian distribution revealing low-bursting and high-bursting cell populations. The mean of the first peak was $22 \%$ spikes in bursts, whereas the second peak was $70 \%$ spikes in bursts.

to regulate DA cell firing by applying specific receptor blockers in the dark (Table 2). Blockade of excitatory glutamatergic input via AMPA/kainate-type or NMDA-type glutamate receptors with $100 \mu \mathrm{M}$ CNQX or $100 \mu \mathrm{M}$ AP-7, respectively, affected neither the firing rate nor the firing pattern of DA cells. However, in control experiments, DA cells indeed responded to application of glutamate or its agonist NMDA in the absence of CNQX or AP-7 (data not shown), suggesting that the lack of effect of glutamatergic inhibitors applied in the dark indicates a lack of excitatory glutamate synaptic input in the dark. In contrast, blockade of inhibitory synaptic inputs with the $\mathrm{GABA}_{\mathrm{A}}$ receptor blocker GABAzine $(20 \mu \mathrm{M})$, or the glycine receptor blocker strychnine $(1 \mu \mathrm{M})$, altered DA cell discharge patterns. Strychnine increased the burst
Table 1. Firing properties of DA cells $(n=92)$

\begin{tabular}{lcc}
\hline & Range & Mean \pm SE \\
\hline Firing rate $(\mathrm{Hz})$ & $1.6-18.5$ & $6.2 \pm 0.3$ \\
Bursting rate $(\mathrm{Hz})$ & $0.03-1.16$ & $0.51 \pm 0.03$ \\
Bursting spikes (\%) & $3.2-93.6$ & $54.6 \pm 2.5$ \\
Time spent bursting (\%) & $0.67-72.22$ & $18.3 \pm 1.4$ \\
Spikes per burst & $3.0-20.0$ & $6.9 \pm 0.4$ \\
Burst duration (ms) & $102-988$ & $332 \pm 17$ \\
Intraburst frequency $(\mathrm{Hz})$ & $7.0-60.9$ & $21.6 \pm 0.9$ \\
\hline
\end{tabular}

duration from $331 \pm 37$ to $457 \pm 39 \mathrm{~ms}(p<0.05 ; n=7)$ and increased the number of spikes per burst as well as the percentage of bursting. Similarly, GABAzine also increased the burst duration of the DA cell spontaneous activity $(366 \pm 37$ in the control vs $438 \pm 40 \mathrm{~ms}$ in the GABAzine; $p<0.05 ; n=7)$. These changes in firing patterns were not accompanied by changes in the overall firing rates of DA cells. Thus, inhibitory synaptic inputs specifically inhibit DA cell bursting in the dark. To further test the influence of synaptic inputs on DA cell activity, we applied a mixture of receptor blockers to simultaneously block the glutamatergic, GABAergic, and glycinergic inputs to DA cells, as well as to block dopamine autoreceptors on the DA cells. Blockade of these known intraretinal synaptic inputs to DA cells caused all cells tested to shift in their firing patterns by markedly increasing bursting while decreasing single spiking (Fig. 2). Again, the overall firing rate was not affected $(5.1 \pm 0.5 \mathrm{~Hz}$ vs $5.6 \pm 1.3 \mathrm{~Hz} ; n=$ $5 ; p>0.05)$, but both the percentage of spikes contained in bursts $(54.5 \pm 5.5 \%$ vs $83.5 \pm 2.1 \% ; n=5 ; p<0.01)$ and the coefficient of variation of spike timing (an indicator of bursting; $1.1 \pm 0.1$ vs $2.8 \pm 0.6 ; n=5 ; p<0.05)$ were increased significantly. These data suggest that the bursting aspect of DA cell spontaneous activity in situ is predominantly generated by a mechanism that is intrinsic to DA cells, which is then modulated by inhibitory synaptic input.

\section{DA cells exhibit two classes of light responses}

Light stimulates dopamine secretion in vertebrate retinas from a wide range of species (Witkovsky, 2004), but the electrophysiological light responses of DA cells and their underlying cellular mechanisms have yet to be defined. We delivered photopic light stimuli consisting of $1 \mathrm{~s}$ flashes of blue $(470 \mathrm{~nm})$, green $(525 \mathrm{~nm})$, or red $(630 \mathrm{~nm})$ light to mouse retinas in darkness and recorded DA cell responses. Figure 3 shows representative examples of the DA cell responses we recorded. Light evoked increases in firing frequency (ON responses) in 28 of $47(60 \%)$ DA cells tested. These $\mathrm{ON}$ responses were present in two classes that differed in temporal dynamics and synaptic input (see below). The majority of ON DA cells (18 of 28) exhibited ON-transient responses consisting of a rapid burst of spikes elicited near the onset of the light stimulus, followed by a decrease in spike frequency, or silence, for the duration of the stimulation. However, a significant minority of ON DA cells ( 10 of 28) exhibited ON-sustained responses, in which the light-evoked increase in action potential frequency was maintained throughout the duration of the light pulse, even for flashes of up to $20 \mathrm{~s}$ in duration (data not shown). Both transient and sustained DA cells were most responsive to $525 \mathrm{~nm}$ light versus $470 \mathrm{~nm}$ light, with greatly reduced responses recorded at $630 \mathrm{~nm}$, suggesting input primarily by midwave cones present in the mouse retina (Nikonov et al., 2006). Despite the anatomical projection of DA cell processes in the OFF sublamina of the inner retina, no OFF responses (increased spiking at the termination of illumination) were recorded in DA cells. As a positive control for 
Table 2. Effects of individual synaptic blockers on spontaneous activity parameters of DA cells

\begin{tabular}{|c|c|c|c|c|c|c|c|}
\hline Group & $\begin{array}{l}\text { Firing rate } \\
(\mathrm{Hz})\end{array}$ & $\begin{array}{l}\text { Burst frequency } \\
(\mathrm{Hz})\end{array}$ & $\begin{array}{l}\text { Bursting } \\
\text { spikes (\%) }\end{array}$ & $\begin{array}{l}\text { Time spent bursting } \\
(\%)\end{array}$ & $\begin{array}{l}\text { Spikes per } \\
\text { burst }\end{array}$ & $\begin{array}{l}\text { Burst duration } \\
\text { (ms) }\end{array}$ & $\begin{array}{l}\text { Intraburst frequency } \\
(\mathrm{Hz})\end{array}$ \\
\hline Control & $6.1 \pm 1.2$ & $0.45 \pm 0.10$ & $57.4 \pm 13.3$ & $15.4 \pm 3.1$ & $7.5 \pm 1.6$ & $343 \pm 46$ & $24.3 \pm 8.5$ \\
\hline $\operatorname{CNQX}(n=4)$ & $6.6 \pm 1.3$ & $0.46 \pm 0.16$ & $47.5 \pm 14.6$ & $14.2 \pm 3.3$ & $6.9 \pm 1.1$ & $340 \pm 48$ & $22.7 \pm 7.1$ \\
\hline Control & $5.5 \pm 1.5$ & $0.35 \pm 0.08$ & $55.3 \pm 19.3$ & $10.6 \pm 3.3$ & $8.2 \pm 4.1$ & $260 \pm 45$ & $28.3 \pm 9.2$ \\
\hline $\operatorname{AP}-7(n=3)$ & $6.7 \pm 2.5$ & $0.40 \pm 0.17$ & $46.4 \pm 21.5$ & $11.9 \pm 5.7$ & $7.5 \pm 3.2$ & $268 \pm 30$ & $26.4 \pm 8.9$ \\
\hline Control & $6.3 \pm 0.7$ & $0.66 \pm 0.07$ & $58.1 \pm 6.3$ & $20.9 \pm 3.7$ & $6.4 \pm 0.5$ & $366 \pm 37$ & $18.3 \pm 1.7$ \\
\hline GABAzine $(n=7)$ & $7.3 \pm 0.6$ & $0.60 \pm 0.04$ & $70.8 \pm 4.0$ & $26.0 \pm 2.0$ & $8.8 \pm 1.4$ & $438 \pm 40^{*}$ & $20.2 \pm 2.4$ \\
\hline Control & $6.5 \pm 0.9$ & $0.54 \pm 0.09$ & $61.5 \pm 9.7$ & $19.4 \pm 4.6$ & $7.6 \pm 1.6$ & $331 \pm 31$ & $21.9 \pm 4.1$ \\
\hline Strychnine $(n=7)$ & $7.9 \pm 1.5$ & $0.53 \pm 0.11$ & $76.1 \pm 9.9^{*}$ & $21.4 \pm 5.6$ & $11.7 \pm 1.7^{*}$ & $457 \pm 39^{*}$ & $24.5 \pm 2.7$ \\
\hline
\end{tabular}

${ }^{*} p<0.05$

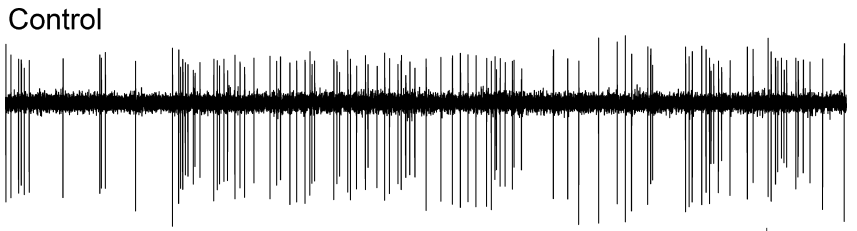

$30 \mathrm{pA}$

Synaptic blocker cocktail

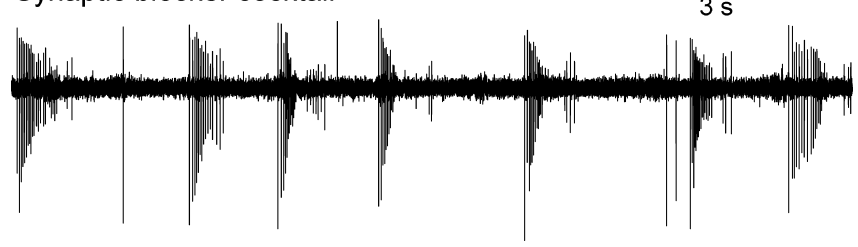

Figure 2. Effects of synaptic blockade on DA neuron spontaneous activity. Synaptic isolation by blockade of intraretinal synaptic inputs to DA neurons with a blocker mixture [containing the following (in $\mu \mathrm{m}$ ): 20 CNQX, 50 AP-5, 30 bicuculline, 100 imidazole-4-acetic acid sodium salt, 3 strychnine, 30 eticlopride] increases bursting. A typical recording of DA neuron spontaneous activity in the absence (top) and presence (bottom) of the blocker mixture is shown.

intact OFF pathway light responses in our retinal preparations, we labeled ganglion cells with red fluorescent DiI by retrograde transport, targeted them for recording using fluorescent snap shot imaging identical to that used to target DA cells, and verified the presence of OFF responses in our retinal preparations (supplemental Fig. 1, available at www.jneurosci.org as supplemental material).

A significant proportion of DA cells (19 of 47, 40\%) was found to be unresponsive to light stimulation at any wavelength tested. Each of these cells was tested with $20 \mathrm{~s}$ light pulses, as well as $1 \mathrm{~s}$ pulses, to test for slow onset kinetics and was still found to be unresponsive to light. These cells exhibited normal spontaneous activity and responded to pharmacological stimulation appropriately (supplemental Fig. 2, available at www.jneurosci.org as supplemental material); thus, there is no indication that they were unhealthy or damaged. In addition, as a positive control for intact light responses in our retinal preparations, we labeled ganglion cells with DiI and targeted them for recording as above. Light responses were intact in $>90 \%$ of ganglion cells recorded in this manner (supplemental Fig. 1, available at www.jneurosci.org as supplemental material), indicating that photoreceptor bleaching or loss of photoreceptor input caused by exposure to imaging light during cell targeting was unlikely to account for more than a small percentage of the unresponsive DA cells. To exclude the possibility that the null light responses of DA cells were attributable to cancellation of antagonistic excitatory and inhibitory inputs, we pharmacologically disrupted the excitatory ON pathway using L-AP-4 (see below) or surround inhibition mediated through the horizontal cell network using the gap junction blocker carbenoxolone. Neither treatment revealed light responses in previously unresponsive DA cells (supplemental Fig. 3 , available at www.jneurosci.org as supplemental material). Thus, the unresponsive DA cells exhibit spontaneous spiking and evidence of synaptic input but not light responses. We suggest that they function in a light-independent manner.

\section{Transient and sustained DA cells have distinct synaptic inputs}

To define the neuronal circuits by which light responses arise in DA cells, we first tested whether light signals from photoreceptors to DA cells pass through ON-type bipolar cells, the primary pathway for transmission of $\mathrm{ON}$ responses from the outer to the inner retina. L-AP-4, an agonist of mGluR6 receptors, is known to block the retinal ON pathway through persistent activation of mGluR6 receptors expressed on ON-bipolar cell dendrites (Slaughter and Miller, 1981). We applied L-AP-4 to 22 lightresponsive DA cells (14 ON-transient DA cells and $8 \mathrm{ON}$ sustained DA cells) and found that light responses were completely blocked by L-AP-4 in all 14 ON-transient DA cells (Fig. $4 A a, A b)$, confirming that this class of DA cell light response involves input from ON bipolar cells. L-AP-4 had no effect on the spontaneous activity of DA cells.

There are two possible pathways by which photoreceptor light signals could reach DA cells through ON-type bipolar cells. One is by direct innervation of DA cells by ON bipolar cells (Hokoc and Mariani, 1987; Boelen et al., 1998), and a second is an indirect pathway through the disinhibition of a serial inhibitory network of GABAergic and/or glycinergic amacrine cells presynaptic to DA cells (Critz and Marc, 1992; Gustincich et al., 1997). To differentiate these possibilities, we recorded light responses of DA cells in the presence of either GABAzine or strychnine and found that robust $\mathrm{ON}$ light responses persisted under the blockade of $\mathrm{GABA}_{\mathrm{A}}$ or glycinergic neurotransmission (Fig. $4 A c, A d$ ). In addition, when GABAzine and strychnine were applied together, light responses persisted in four of five DA cells. These results indicate that ON bipolar cells do not require a serial inhibitory network to activate DA cells. Instead, DA cells most likely receive direct inputs from ON bipolar cells. Additional evidence for mediation of the transient on response by glutamatergic neurons, such as $\mathrm{ON}$ bipolar cells, was provided by application of specific glutamate receptor blockers (Fig. $4 \mathrm{~B}$ ). We found that CNQX completely blocked light-evoked ON-transient responses of DA cells (Fig. $4 B b$ ), whereas AP-7 had no effect (Fig. $4 B d$ ), indicating that ON bipolar cell input to DA cells is mediated by AMPA and/or kainite-type glutamate receptors.

In contrast to the complete blockade by L-AP-4 of light re- 


\section{ON-transient}

$470 \mathrm{~nm}$

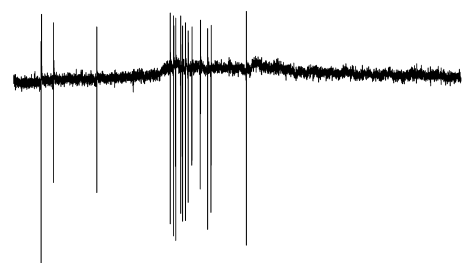

$525 \mathrm{~nm}$

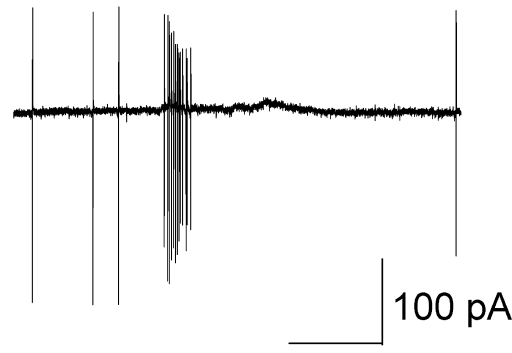

$1 \mathrm{~s}$

$630 \mathrm{~nm}$
ON-sustained
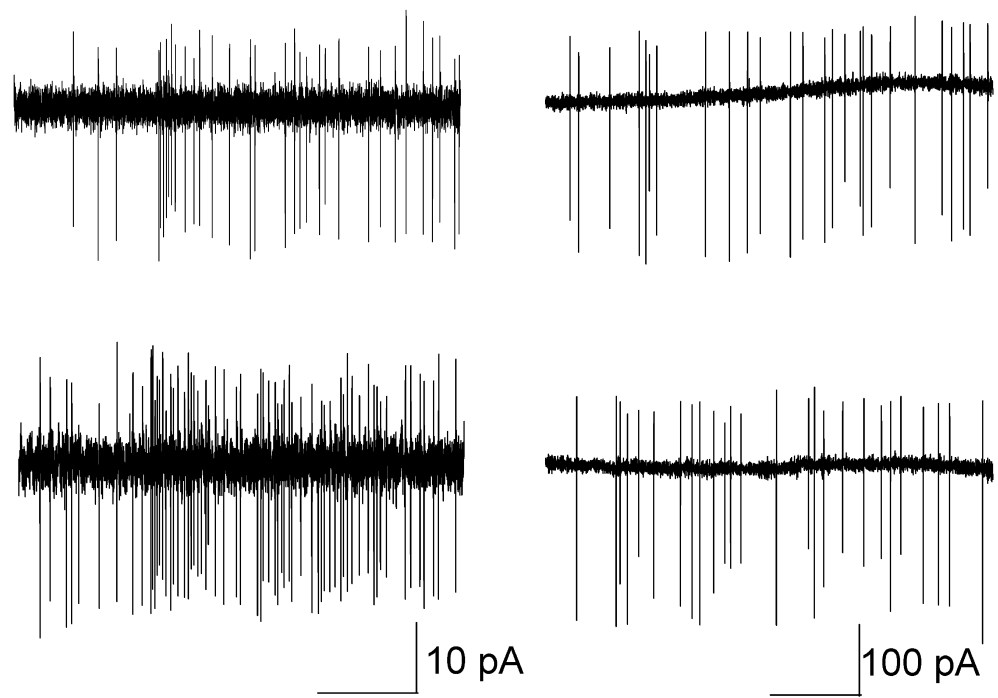

$1 \mathrm{~s}$

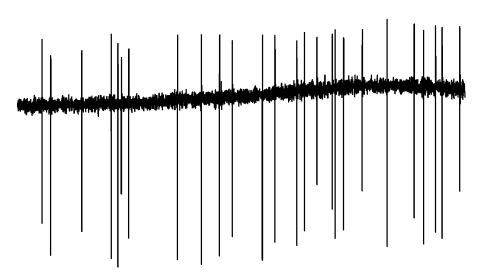

Light-independent
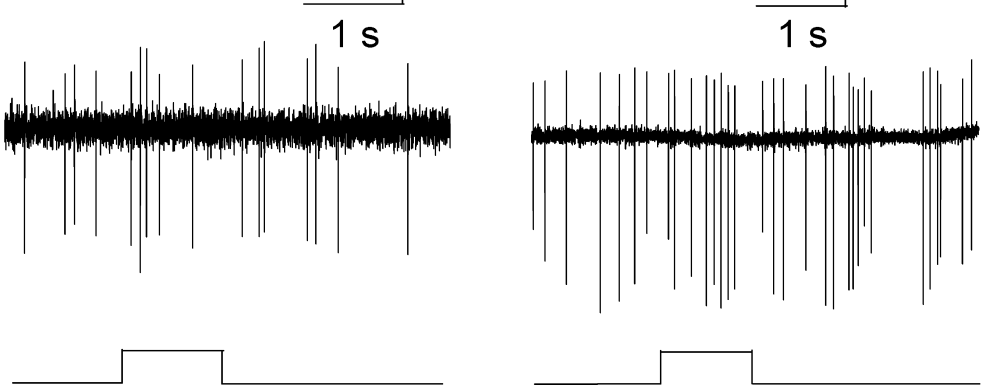

Figure 3. Light responses of DA neurons. Typical recordings of DA neuron light responses to $1 \mathrm{~s}$ flashes (square waves below response traces) of the indicated wavelength are shown. Each column shows responses to different wavelengths of an individual neuron..

sponses in ON-transient DA cells, L-AP-4 did not block light responses in seven of eight $\mathrm{ON}$-sustained DA cells. Figure 5 shows a typical recording in which the $\mathrm{ON}$-sustained light response is undiminished in the presence of 10 or $30 \mu \mathrm{M} \mathrm{L}-\mathrm{AP}-4$ (Fig. $5 a-c$ ). Increasing the L-AP- 4 concentration to $100 \mu \mathrm{M}$, a concentration that completely blocked $\mathrm{ON}$ bipolar cell responses in the mouse retina (Protti et al., 2005) and that in our preparations completely blocked ON components of light responses in ganglion cells (supplemental Fig. 1, available at www.jneurosci.org as supplemental material), failed to diminish light responses in sustained DA cells, also suggesting that sustained light responses are not mediated by $\mathrm{ON}$ bipolar cell input. One possible pathway by which L-AP-4-resistant ON responses could arise in DA cells is through indirect input from OFF bipolar cells through inhibitory amacrine cells. To test this possibility, GABAzine or strychnine were used to block $\mathrm{GABA}_{\mathrm{A}}$ receptors and glycine receptors. Neither individual treatment blocked $\mathrm{ON}$-sustained responses of DA cells (Fig. $5 d, e)$, nor did their combined application $(n=2)$, thus excluding the possibility that L-AP-4-resistant light responses are mediated by inhibition of the OFF pathway. However, when CNQX was applied, ON-sustained responses were completely blocked (Fig. $5 f$ ), indicating that glutamatergic excitatory synaptic transmission, from an as yet unidentified source, is involved in the neuronal circuit generating $\mathrm{ON}$-sustained responses of DA cells.

\section{Discussion}

Fluorescent labeling of dopaminergic neurons with a genetically encoded RFP reporter construct has enabled targeted electro- physiological recordings of these neurons in intact retinal circuits and characterization of their spontaneous activity, synaptic inputs, and light responses. Our findings indicate that, in situ, retinal dopaminergic amacrine neurons are spontaneously bursting neurons and that their intrinsic bursting mechanism is blunted by tonic inhibitory synaptic inputs in the dark. Furthermore, DA neurons exhibit diversity in their spike patterning, with distinct high- and low-bursting populations, and in their light responses, producing transient and sustained $\mathrm{ON}$ responses mediated by distinct synaptic mechanisms.

\section{DA cell spontaneous activity and regulation}

Dopaminergic amacrine cells in the intact isolated retina generate spontaneous bursting spike patterns that are enhanced by synaptic blockade. The intrinsic bursting exhibited by DA cells in situ contrasts with the spike patterns observed in acutely isolated and dispersed retinal DA cells, which exhibit spontaneous regular single spikes but do not burst (Feigenspan et al., 1998). A number of factors may explain the differences in results, including the loss of proximal dendrites, alteration of ion channel complement or changes in membrane properties during physical isolation of DA cells in the former studies (Feigenspan et al., 1998), or the action of an unknown neurotransmitter on synaptically isolated cells in situ in the present study.

To reveal intrinsic bursting, we have blocked the known intraretinal glutamatergic, GABAergic, and glycinergic synaptic inputs to DA cells, as well as dopamine autoreceptors on DA neurons themselves (Gustincich et al., 1997). These procedures would have also blocked any input to DA neurons from cholin- 
A

a: Control

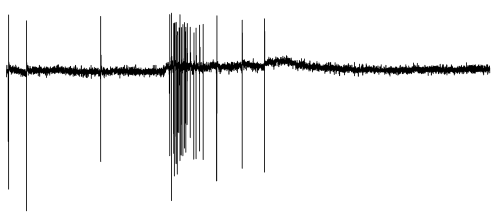

b: $10 \mu \mathrm{M} \mathrm{L-AP4}$
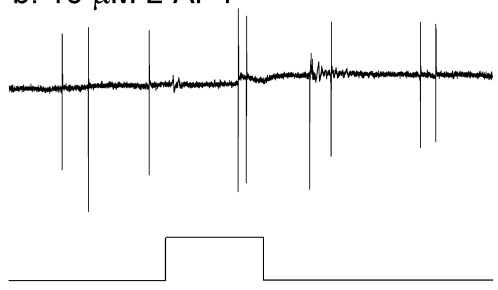

B

a: Control

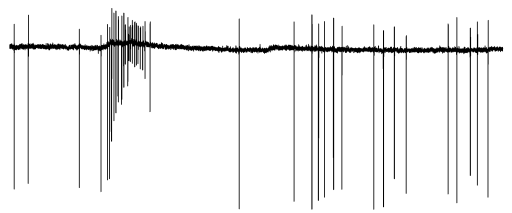

b: $100 \mu \mathrm{M} C N Q X$

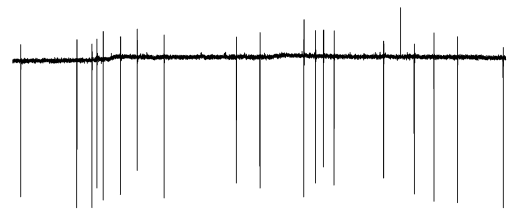

$\square$

\section{c: $20 \mu \mathrm{M}$ GABAzine}

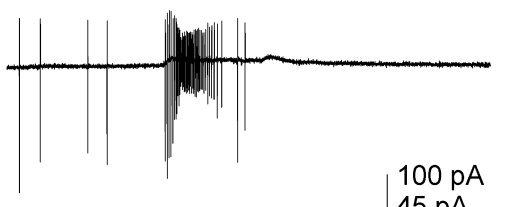

$\mathrm{d}: 1 \mu \mathrm{M}$ Strychnine
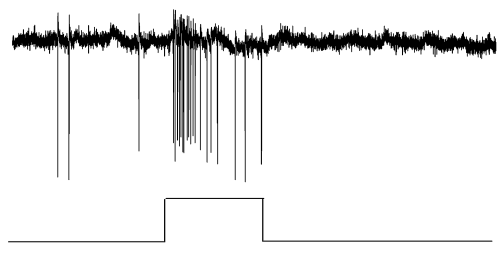

c: Washout

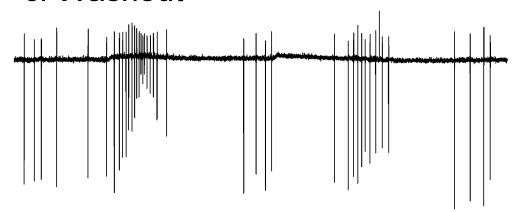

$40 \mathrm{pA}$
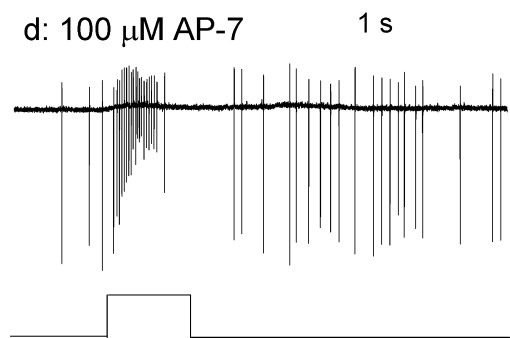

Figure 4. Effects of synaptic blockers on ON-transient DA neuron responses. $A, 0 \mathrm{~N}$-transient DA neuron light responses $(\boldsymbol{A a}$ $525 \mathrm{~nm})$ were blocked by the $\mathrm{ON}$-channel blocker L-AP-4 $(\boldsymbol{A} \boldsymbol{b} ; n=14)$ but were insensitive to GABAzine $(A \boldsymbol{c} ; n=8)$ and strychnine $(\boldsymbol{A d} ; n=5) . \boldsymbol{B}, 0 \mathrm{~N}$-transient DA neuron responses (Ba) were reversibly blocked by $\mathrm{CNQX}(\boldsymbol{B} \boldsymbol{b}, \boldsymbol{B} \boldsymbol{c} ; n=6)$, but unaffected by AP-7 $(\boldsymbol{B d} ; n=5)$. Flashes are indicated by square waves below response traces.

ergic amacrine cells, which is indirectly mediated through GABA (Neal et al., 2001). DA cells also receive histaminergic input from centrifugal fibers in monkey and rat retinas (Gastinger et al., 2006), but this extraretinal input, if it exists in the mouse, was interrupted in our isolated retinas. The retinal neurohormone melatonin is a modulator of dopamine release (Dubocovich, 1983), but the mouse strain we used (C57BL/6J) lacks the ability to synthesize melatonin because of genetic mutations (Ebihara et al., 1986). Electrical coupling through gap junctions is unlikely to contribute to spike regulation in DA cells, because these lack discernable tracer coupling (Zhang et al., 2004), and the gap junction blocker carbenoxolone does not affect spontaneous activity (supplemental Fig. 3, available at www.jneurosci.org as supplemental material). Thus, although we cannot exclude all possible synaptic or humoral mechanisms, a parsimonious explanation of our findings is that bursting is intrinsically generated in DA cells in situ. Similar mixed spiking/bursting patterns are observed in brain dopamine neurons in vivo, although the source of their bursting is thought to be synaptic (Kitai et al., 1999). Both in vivo and in vitro experiments demonstrate that retinal DA cells are strongly biased toward spontaneous neural activity, the pattern and rate of which is then modulated by specific synaptic inputs.

In the absence of light stimulation, the bursting component of DA cell spontaneous activity is reduced by glycinergic and GABAergic synaptic input. In contrast, we found no evidence of excitatory glutamatergic input in the dark. Although a variety of factors including neurotransmitter synthesis and metabolism contribute to the regulation of dopamine release, bursting spike patterns are clearly associated with enhanced dopamine release from dopaminergic neurons in the brain (Gonon, 1988; Floresco et al., 2003). Thus, by reducing intrinsic DA neuron bursting, tonic glycinergic and GABAergic input likely serves to reduce the tonic level of dopamine release in the dark. These results, along with observations of increased retinal dopamine release in the dark in the presence of GABA or glycine antagonists (Kamp and Morgan, 1981; Morgan and Kamp, 1983), demonstrate that inhibitory inputs from GABAergic and glycinergic amacrine cells are primary regulators of DA neuron activity in the dark. Furthermore, the existence of distinct high- and low-bursting populations of DA cells, which are uniformly converted to high bursting through synaptic blockade, suggests heterogeneity in the degree of synaptic regulation of intrinsic bursting activity across DA cells.

\section{DA cell light responses}

DA cell light responses reveal three distinct functional outputs from the DA neuron population: ON-transient, ON-sustained, and light-independent neural activity. ON-transient DA cells are excited by ON bipolar cells, whereas ON-sustained DA cells receive an excitatory glutamatergic synaptic input from a source not yet identified. Although ON pathways make clear contributions to the light responses of DA cells, we found no evidence for OFF pathway contributions, either in the form of direct OFF responses or as a disinhibitory component of ON responses. Thus, our results do not support the previous proposal that disinhibition of OFF-driven GABAergic amacrine input is a principal mechanism of DA cell light responses (Critz and Marc, 1992; Gustincich et al., 1997). The predominance of physiologically defined ON pathway input and apparent lack of OFF pathway input to DA cells is surprising, given the ramification of DA cell processes in the outer portion of the retinal inner plexiform layer (IPL) where OFF synapses are made. This pattern of physiological and anatomical synaptic inputs is not unprecedented, because primate intrinsically photoreceptive ganglion cells show similar OFF sublamina process branching, yet have functional $O N$ pathway input (Dacey et al., 2005). Indeed, there are examples of sparse bipolar cell contacts to DA cells in the ON sublamina of the IPL, both in the literature (Kolb et al., 1990) and in our retinal preparations (supplemental Fig. 4, available at www.jneurosci. 
org as supplemental material). These synapses apparently drive the $\sim 40 \%$ of DA cells that demonstrate ON-transient responses. ON pathway input to DA cells is likely a general feature of vertebrate retinas, because L-AP-4 blocks light-induced dopamine release in monkey and frog retinas (Boatright et al., 1994; Boelen et al., 1998).

In addition to the transient $\mathrm{ON}$ responses mediated by $\mathrm{ON}$ bipolar cell input, we also observed L-AP-4-resistant sustained $\mathrm{ON}$ responses in DA cells. Bistratified bipolar cells make excitatory contacts to DA cells in cat and monkey retinas (Hokoc and Mariani, 1987; Kolb et al., 1990), and in fish retinas, bistratified bipolar cells exhibit L-AP-4-resistant ON light responses mediated by the glutamate-chloride cotransporter EAAT5 (Wong et al., 2005). However, in the mouse retina, EAAT5 localization and function differs from the fish because it is limited to rod, cone, and rod bipolar cell terminals, where it acts primarily to negatively regulate synaptic glutamate release and clear released glutamate (Hasegawa et al., 2006; Wersinger et al., 2006). Application of the transporter inhibitor TBOA causes loss of DA cell spontaneous activity as well as loss of both ON-transient and ON-sustained light responses, effects that are blocked by the glutamate receptor inhibitor CNQX (data not shown). These actions are consistent with the role of EAAT5 in limiting glutamate release and mediating its clearance from photoreceptor and bipolar cell synapses, and are inconsistent with EAAT5 as a specific mediator of L-AP-4-resistant ON responses in mouse DA cells. Recently, L-AP-4-resistant ON responses have also been described in mouse retinal ganglion cells where they were attributed to ON responses carried in the OFF channel when the ON channel is inhibited (Renteria et al., 2006). The L-AP-4-resistant responses reported in ganglion cells were of substantially longer latency than DA cell L-AP-4-resistant ON responses and were transient rather than sustained. They were not evident before application of L-AP-4 and only emerged in retinal ganglion cells with demonstrated OFF light responses, again, unlike DA cell-sustained responses. Thus, the L-AP-4-resistant light responses in mouse DA cells and ganglion cells show substantially different characteristics. Additional experiments are necessary to understand the mechanisms of L-AP-4-resistant $\mathrm{ON}$ responses in retinal neurons.

\section{DA cell heterogeneity and dopaminergic function}

Our results provide an expanded basis for understanding the broad range of experimental results obtained in previous studies of the retinal dopaminergic system. They demonstrate distinct functional modes of DA cell spike activity regulation in the dark and in the light that can subserve the observed sustained, transient, and light-independent circadian modes of dopamine release (Bauer et al., 1980; Mangel and Dowling, 1985; Umino et al., 1991; Weiler et al., 1997; Nir et al., 2000; Doyle et al., 2002a,b; Ribelayga et al., 2004) and suggest that retinal dopaminergic neurons comprise functionally distinct subpopulations tuned to sus-

\section{d: $20 \mu$ M GABAzine}

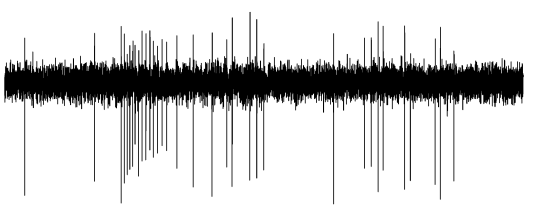

e: $1 \mu \mathrm{M}$ strychnine

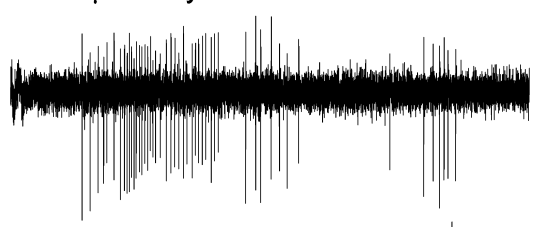

$10 \mathrm{pA}$

f: $100 \mu \mathrm{M} C N Q X$ $20 \mathrm{pA}$
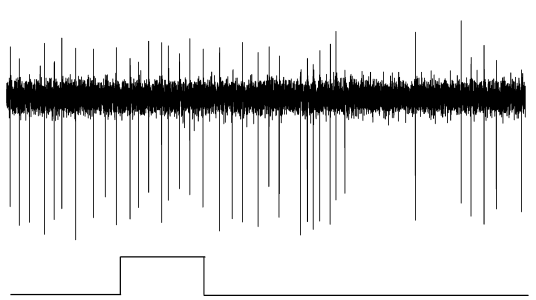

Figure 5. Effects of synaptic blockers on $0 \mathrm{~N}$-sustained DA neuron responses. ON-sustained DA neuron light responses ( $\boldsymbol{a} ; 525$ nm) were unaffected by L-AP-4 (b, $; n=5$ of 6$),$ GABAzine $(\boldsymbol{d} ; n=3)$, and strychnine $(\boldsymbol{e} ; n=3)$ but were reversibly blocked by CNQX $(\boldsymbol{f} ; n=3)$. Calibration: $\boldsymbol{a}-\boldsymbol{c}, 20 \mathrm{pA}, 1 \mathrm{~s} ; \boldsymbol{d}-\boldsymbol{f}, 10 \mathrm{pA}, 1 \mathrm{~s}$. Flashes are indicated by square waves below response traces.

tained versus transient illumination or regulated by intracellular circadian clock genes (Ruan et al., 2006). Variations in the precise balance of these mechanisms likely account for the range of experimental results previously observed across adaptation conditions and species.

The striking functional heterogeneity of DA amacrine cells revealed in their spontaneous activity and light responses is part of an emerging body of evidence that suggests that dopaminergic neurons throughout the CNS are more heterogeneous than previously appreciated. Recently, dopaminergic neurons in the ventral tegmental area were found to exhibit four distinct classes of spiking patterns that were modulated differentially by pharmacological inputs (Mameli-Engvall et al., 2006). In addition, midbrain dopaminergic neurons exhibit substantial neuropeptide phenotype heterogeneity (Seutin, 2005), as do retinal DA neurons for gene expression (Ruan et al., 2006). The precise extent to which distinct functional output groups represent intrinsic subpopulations of dopaminergic cells versus distinct functional states driven by synaptic input is unknown at this point. Additional investigation of the accessible retinal DA neurons is likely to reveal the basis for functional complexity in dopaminergic neuron populations and increase our understanding of the roles of these neurons in normal neural function and in neurological disease.

\section{References}

Bauer B, Ehinger B, Aberg L (1980) [3H]-dopamine release from the rabbit retina. Albrecht Von Graefes Arch Klin Exp Ophthalmol 215:71-78.

Boatright JH, Gordon JR, Iuvone PM (1994) Inhibition of endogenous dopamine release in amphibian retina by L-2-amino-4-phosphonobutyric acid (L-AP4) and trans-2-aminocyclopentane-1,3-dicarboxylate (ACPD). Brain Res 649:339-342.

Boelen MK, Boelen MG, Marshak DW (1998) Light-stimulated release of 
dopamine from the primate retina is blocked by 1-2-amino-4phosphonobutyric acid (APB). Vis Neurosci 15:97-103.

Critz SD, Marc RE (1992) Glutamate antagonists that block hyperpolarizing bipolar cells increase the release of dopamine from turtle retina. Vis Neurosci 9:271-278.

Dacey DM, Liao HW, Peterson BB, Robinson FR, Smith VC, Pokorny J, Yau KW, Gamlin PD (2005) Melanopsin-expressing ganglion cells in primate retina signal colour and irradiance and project to the LGN. Nature 433:749-754.

Dowling JE, Ehinger B (1975) Synaptic organization of the aminecontaining interplexiform cells of the goldfish and Cebus monkey retinas. Science 188:270-273.

Doyle SE, Grace MS, McIvor W, Menaker M (2002a) Circadian rhythms of dopamine in mouse retina: the role of melatonin. Vis Neurosci 19:593-601.

Doyle SE, McIvor WE, Menaker M (2002b) Circadian rhythmicity in dopamine content of mammalian retina: role of the photoreceptors. J Neurochem 83:211-219.

Dubocovich ML (1983) Melatonin is a potent modulator of dopamine release in the retina. Nature 306:782-784.

Ebihara S, Marks T, Hudson DJ, Menaker M (1986) Genetic control of melatonin synthesis in the pineal gland of the mouse. Science 231:491-493.

Feigenspan A, Gustincich S, Bean BP, Raviola E (1998) Spontaneous activity of solitary dopaminergic cells of the retina. J Neurosci 18:6776-6789.

Floresco SB, West AR, Ash B, Moore H, Grace AA (2003) Afferent modulation of dopamine neuron firing differentially regulates tonic and phasic dopamine transmission. Nat Neurosci 6:968-973.

Gastinger MJ, Barber AJ, Vardi N, Marshak DW (2006) Histamine receptors in mammalian retinas. J Comp Neurol 495:658-667.

Gonon FG (1988) Nonlinear relationship between impulse flow and dopamine released by rat midbrain dopaminergic neurons as studied by in vivo electrochemistry. Neuroscience 24:19-28.

Grace AA, Bunney BS (1984) The control of firing pattern in nigral dopamine neurons: burst firing. J Neurosci 4:2877-2890.

Gustincich S, Feigenspan A, Wu DK, Koopman LJ, Raviola E (1997) Control of dopamine release in the retina: a transgenic approach to neural networks. Neuron 18:723-736.

Gustincich S, Feigenspan A, Sieghart W, Raviola E (1999) Composition of the $\mathrm{GABA}_{\mathrm{A}}$ receptors of retinal dopaminergic neurons. J Neurosci 19:7812-7822.

Hasegawa J, Obara T, Tanaka K, Tachibana M (2006) High-density presynaptic transporters are required for glutamate removal from the first visual synapse. Neuron 50:63-74.

Hokoc JN, Mariani AP (1987) Tyrosine hydroxylase immunoreactivity in the rhesus monkey retina reveals synapses from bipolar cells to dopaminergic amacrine cells. J Neurosci 7:2785-2793.

Kamp CW, Morgan WW (1981) GABA antagonists enhance dopamine turnover in the rat retina in vivo. Eur J Pharmacol 69:273-279.

Kitai ST, Shepard PD, Callaway JC, Scroggs R (1999) Afferent modulation of dopamine neuron firing patterns. Curr Opin Neurobiol 9:690-697.

Kolb H, Cuenca N, Wang HH, Dekorver L (1990) The synaptic organization of the dopaminergic amacrine cell in the cat retina. J Neurocytol 19:343-366.
Mameli-Engvall M, Evrard A, Pons S, Maskos U, Svensson TH, Changeux JP, Faure P (2006) Hierarchical control of dopamine neuron-firing patterns by nicotinic receptors. Neuron 50:911-921.

Mangel SC, Dowling JE (1985) Responsiveness and receptive field size of carp horizontal cells are reduced by prolonged darkness and dopamine. Science 229:1107-1109.

Morgan WW, Kamp CW (1983) Effect of strychnine and of bicuculline on dopamine synthesis in retinas of dark-maintained rats. Brain Res 278:362-365.

Neal MJ, Cunningham JR, Matthews KL (2001) Activation of nicotinic receptors on GABAergic amacrine cells in the rabbit retina indirectly stimulates dopamine release. Vis Neurosci 18:55-64.

Nikonov SS, Kholodenko R, Lem J, Pugh Jr EN (2006) Physiological features of the S- and M-cone photoreceptors of wild-type mice from singlecell recordings. J Gen Physiol 127:359-374.

Nir I, Haque R, Iuvone PM (2000) Diurnal metabolism of dopamine in the mouse retina. Brain Res 870:118-125.

Protti DA, Flores-Herr N, Li W, Massey SC, Wassle H (2005) Light signaling in scotopic conditions in the rabbit, mouse and rat retina: a physiological and anatomical study. J Neurophysiol 93:3479-3488.

Puopolo M, Hochstetler SE, Gustincich S, Wightman RM, Raviola E (2001) Extrasynaptic release of dopamine in a retinal neuron: activity dependence and transmitter modulation. Neuron 30:211-225.

Renteria RC, Tian N, Cang J, Nakanishi S, Stryker MP, Copenhagen DR (2006) Intrinsic ON responses of the retinal OFF pathway are suppressed by the ON pathway. J Neurosci 26:11857-11869.

Ribelayga C, Wang Y, Mangel SC (2004) A circadian clock in the fish retina regulates dopamine release via activation of melatonin receptors. J Physiol (Lond) 554:467-482.

Ruan GX, Zhang DQ, Zhou T, Yamazaki S, McMahon DG (2006) Circadian organization of the mammalian retina. Proc Natl Acad Sci USA 103:9703-8.

Seutin V (2005) Dopaminergic neurones: much more than dopamine? Br J Pharmacol 146:167-169.

Slaughter MM, Miller RF (1981) 2-Amino-4-phosphonobutyric acid: a new pharmacological tool for retina research. Science 211:182-185.

Umino O, Lee Y, Dowling JE (1991) Effects of light stimuli on the release of dopamine from interplexiform cells in the white perch retina. Vis Neurosci 7:451-458.

Weiler R, Baldridge WH, Mangel SC, Dowling JE (1997) Modulation of endogenous dopamine release in the fish retina by light and prolonged darkness. Vis Neurosci 14:351-356.

Wersinger E, Schwab Y, Sahel JA, Rendon A, Pow DV, Picaud S, Roux MJ (2006) The glutamate transporter EAAT5 works as a presynaptic receptor in mouse rod bipolar cells. J Physiol (Lond) 577:221-234.

Witkovsky P (2004) Dopamine and retinal function. Doc Ophthalmol 108:17-40.

Wong KY, Cohen ED, Dowling JE (2005) Retinal bipolar cell input mechanisms in giant danio. II. Patch-clamp analysis of on bipolar cells. J Neurophysiol 93:94-107.

Zhang DQ, Stone JF, Zhou T, Ohta H, McMahon DG (2004) Characterization of genetically labeled catecholamine neurons in the mouse retina. NeuroReport 15:1761-1765. 